\title{
Studies on the Bistability of a Dissipative Medium Inside a Resonator
}

\author{
M. M. EL-Nicklawy, A.F. Hassan, A. T. Matar, A. A. Hemeda, \\ and A. I. Ali \\ Physics Department, Faculty of Science, Helwan University, \\ Cairo, Egypt
}

Through the differential equation describing the behavior of the nonlinear polarization of a medium with respect to the incident field, the Maxwell field equations and the boundary conditions of the field inside a resonator. Relation between the output field and the incident field, describing bistability phenomena, is obtained.The effect of the spectral profile of the incident field and its spectral half width on the bistability phenomena is also studied.

\section{Introduction:}

The high power densities available in coherent laser beams have made possible the experimental observation of nonlinear effects of a medium. Franken and Co-workers [1] demonstrated second-harmonic generation of light, Kaiser and Garrett [2] two-photon absorption, Giordmaine [3] mixing of light beams and Woodburg discovered stimulated Raman scattering [4] and Maker and Co-workers [5] demonstrated also third harmonic generation.

Nonlinear optical effects may be also used to make all optical switches. The optical phase modulation in the Kerr medium may be converted into intensity modulation by placing the medium in one leg of an interferometer, so that as the control light is turned on and off, the transmittance of the interferometer is switched between 1 and 0 .

A bistable (or two-state) system has an output that can take only one of the two distinct stable values, no matter what input is applied. Switching between these values may be achieved by a temporary change of the level of the input. Bistable devices are important in the digital circuits and in communications, signal processing, and computing. They are used as switches, logic gates and memory elements (flip-flops) [6]. 
Two features are required for making a bistable device: nonlinearity and feedback. If the output of a nonlinear optical medium is fed back by optical resonator and used to control the transmission of light through the medium itself, bistable behavior can be exhibited.

A Fabry-Perot interferometer containing a nonlinear dielectric medium that can be regarded as producing an intensity dependent refractive index and absorption coefficient can become bistable or multi-stable at sufficient high incident intensity [7-11].

Here the bistability phenomenon is described from an interferometric point of view through the nonlinearity of the refractive index and the absorption coefficient of a dielectric medium. The effects of the spectral half width of the deriving wave and the finess of a Fabry-Perot resonator on the phenomenon are represented.

\section{Theory:}

The properties of a dielectric medium through which an electromagnetic wave propagates are completely described by the relation between the polarization density vector and the electric field vector. The mathematical relation between them defines the system and is governed by the characteristics of the medium. The medium is said to be nonlinear if this relation is nonlinear.

Consider a dielectric medium for which the dynamic relation between the polarization density $\mathrm{P}$ and the electric field $\mathrm{E}$ is described by the following nonlinear second order differential equation,

$$
\frac{d^{2} P}{d t^{2}}+2 \sigma \frac{d P}{d t}+\omega_{o}^{2} P+\alpha P^{2}+\beta P^{3}=\gamma E(z, t)
$$

with $\quad E(z, t)=\frac{1}{2}\left\{\varepsilon_{\omega}(z) \exp i(\omega t-k z)+C C\right\}$

where: $\quad \mathrm{P}=\mathrm{Nex}, \alpha=\frac{a}{N e}, \quad \beta=\frac{b}{(N e)^{2}}, \quad \gamma=\frac{N e^{2}}{m}$

$\mathrm{N}$ is the atom density

$E$ is the electron charge

$\mathrm{M}$ is the electron mass

$\omega_{0}$ is the natural oscillation frequency of the dipole 
$\sigma \quad$ is the damping coefficient of the dipole

$\mathrm{a}, \mathrm{b}$ are material constants

ex is the dipole moment

Representing the polarization density as a function of space and time as follows:

$$
\begin{aligned}
P(z, t)= & P_{o}(z)+\frac{1}{2}\left\{P_{\omega}(z) \exp i(\omega t-k z)+C C\right\}+\frac{1}{2}\left\{P_{2 \omega}(z) \exp 2 i(\omega t-k z)+C C\right\} \\
& +\frac{1}{2}\left\{P_{3 \omega}(z) \exp 3 i(\omega t-k z)+C C\right\}
\end{aligned}
$$

where:

$$
P_{o}(z), P_{\omega}(z), P_{2 \omega}(z) \text { and } P_{3 \omega}(z) \text { are to be determined }
$$

As a first approximation, the terms $\alpha P^{2}$ and $\beta P^{3}$ in equation (1) were neglected, yielding:

$$
\frac{d^{2} P}{d t^{2}}+2 \sigma \frac{d P}{d t}+\omega_{o}^{2} P=\frac{1}{2} \gamma E(z, t)
$$

The solution of Eq.(4) is:

$$
P_{1}(z, t)=\frac{\gamma}{\left[\left(\omega_{0}^{2}-\omega^{2}\right)^{2}+4 \sigma^{2} \omega^{2}\right]^{\frac{1}{2}}} \exp (i \theta) E(z, t)=\chi E(z, t)
$$

where:

$$
\chi=\frac{\gamma}{\left[\left(\omega_{0}^{2}-\omega^{2}\right)^{2}+4 \sigma^{2} \omega^{2}\right]^{\frac{1}{2}}} \exp (i \theta)
$$

and

$$
\tan \theta=\frac{2 \sigma \omega}{\omega_{0}^{2}-\omega^{2}}
$$

Substituting for $\alpha P^{2}$ and $\beta P^{3}$ in equation (1) by $\mathrm{P}_{1}(\mathrm{z}, \mathrm{t})$, we get :

$$
\frac{d^{2} P}{d t^{2}}+2 \sigma \frac{d P}{d t}+\omega_{o}^{2} P=\gamma E(z, t)-\alpha \chi^{2} E^{2}(z, t)+\beta \chi^{3} E^{3}(z, t)
$$


Employing Eq.(3) on Eq.(7), we get:

$$
P_{\omega}(z)=\frac{\gamma-\frac{3}{4} \beta \chi^{3}|\varepsilon(z)|^{2}}{\omega_{0}^{2}-\omega^{2}+2 i \sigma \omega} \varepsilon(z)
$$

The present work is restricted on the frequency $\omega$ of the deriving field, therefore we are concerned only with $P_{\omega}(z)$. Equation (8) can be written in the form

$$
P_{\omega}(z)=\left\{\Gamma \exp (i \theta)-\frac{\frac{3}{4} \beta|\varepsilon(z)|^{2} \Gamma^{4}}{\gamma} \exp (2 i \theta)\right\} \varepsilon(z)
$$

where

$$
\Gamma=\frac{\gamma}{\left[\left(\omega_{0}^{2}-\omega^{2}\right)^{2}+4 \sigma^{2} \omega^{2}\right]^{\frac{1}{2}}}
$$

Now setting the medium inside A fabry-Perot resonator and assume that the amplitude $\varepsilon(z)$ various slowly compared with the carrier wave expi( $\omega t-k z)$, this justifies the following inequalities,

$$
\left|\frac{d \varepsilon(z)}{d z}\right| \ll k|\mathcal{E}(z)| \text { and } \quad\left|\frac{d^{2} \varepsilon(z)}{d z^{2}}\right|<k\left|\frac{d \varepsilon(z)}{d z}\right|
$$

The forward and backward field equations inside the resonator under the previous inequalities can be deduced through Maxwell equation in the medium in the form,

$$
\begin{aligned}
& \frac{\partial E_{F}(z, t)}{\partial t}+C \frac{\partial E_{F}(z, t)}{\partial z}=-\frac{i \omega}{2 \varepsilon_{0}} P_{F}(z, t) \\
& \frac{\partial E_{B}(z, t)}{\partial t}-C \frac{\partial E_{B}(z, t)}{\partial z}=\frac{i \omega}{2 \varepsilon_{0}} P_{B}(z, t)
\end{aligned}
$$

Where:

$$
\begin{aligned}
& E_{F}(z, t)=\frac{1}{2}\left\{\varepsilon_{F}(z) \exp i(\omega t-k z)+C C\right\} \\
& E_{B}(z, t)=\frac{1}{2}\left\{\varepsilon_{B}(z) \exp i(\omega t-k(z-L))+C C\right\}
\end{aligned}
$$




$$
\begin{aligned}
& P_{F}(z, t)=\frac{1}{2}\left\{P_{F}(z) \exp i(\omega t-k z)+C C\right\} \\
& P_{B}(z, t)=\frac{1}{2}\left\{P_{B}(z) \exp i(\omega t-k(z-L))+C C\right\}
\end{aligned}
$$

Analogy to Eqn. (9), we can consider:

$$
\begin{aligned}
& P_{F}(z)=\left\{\Gamma \exp (i \theta)-\frac{\frac{3}{4} \beta|\varepsilon(z)|^{2} \Gamma^{4}}{\gamma} \exp (2 i \theta)\right\} \varepsilon_{F}(z) \\
& P_{B}(z)=\left\{\Gamma \exp (i \theta)-\frac{\frac{3}{4} \beta|\varepsilon(z)|^{2} \Gamma^{4}}{\gamma} \exp (2 i \theta)\right\} \varepsilon_{B}(z)
\end{aligned}
$$

To solve the forward field equation (11) through equations (13), (15), and (17), we firstly consider that $\mathrm{P}_{F}(z)=\Gamma \exp (i \theta) \varepsilon_{F}(z)$ and neglecting the nonlinear term which depends on $\left|\varepsilon_{F}(Z)\right|^{2}$. Thus,

$$
\frac{\partial \varepsilon_{\omega}^{F}}{\partial z}=-\frac{i \omega}{2 \varepsilon_{0} C} \Gamma \exp (i \theta) \varepsilon_{F}(z)
$$

The solution of this Eqn. can be given in the form,

$$
\varepsilon_{\omega}^{F}(z)=\varepsilon_{F}(0) \exp \left(-\frac{i \omega \Gamma}{2 \varepsilon_{0} C}(\cos \theta+i \sin \theta) z\right)
$$

the above equation gives,

$$
\left|\varepsilon_{F}(z)\right|^{2}=\left|\varepsilon_{F}(0)\right|^{2} \exp \left(-\frac{\omega \Gamma \sin \theta}{\varepsilon_{0} C} z\right)
$$

Now substitute for $\left|\varepsilon_{F}(z)\right|^{2}$ into Eqn. (17), it follows,

$$
\mathrm{P}_{\mathrm{F}}(\mathrm{z})=\left(\Lambda-D \exp \left(-\frac{\omega \Gamma \sin \theta}{\varepsilon_{0} C} z\right)\right) \varepsilon_{F}(z)
$$


where $\quad \Lambda=\Gamma \exp (i \theta), \quad \mathrm{D}=\frac{\frac{3}{4} \beta\left|\mathcal{E}_{F}(0)\right|^{2} \Gamma^{4}}{\gamma} \exp (2 i \theta)$

Now solving the forward field, Eq.(11), after considering equations (13), (15), (20) we get ;

$$
\frac{\partial \varepsilon_{\omega}^{F}}{\partial z}=-\frac{i \omega}{2 \varepsilon_{0} C}\left(\Lambda-D \exp \left(-\frac{\omega \Gamma \sin \theta}{\varepsilon_{0} C} z\right)\right) \varepsilon_{F}(z)
$$

Considering Eqn. (21), the solution of Eqn. (22) is:

$$
\begin{aligned}
\ln \left(\frac{\mathcal{E}_{F}(z)}{\varepsilon_{F}(0)}=\right. & -\frac{i \omega}{2 \varepsilon_{0} C} \Gamma \cos \theta Z+\frac{\omega}{2 \varepsilon_{0} C} \Gamma \sin \theta Z-i \frac{d_{F}}{2 \Gamma \sin \theta} \exp \left(\frac{-\omega \Gamma \sin \theta}{z_{o} C} z\right) \cos 2 \theta \\
& +\frac{d_{F}}{2 \Gamma \sin \theta} \exp \left(\frac{-\omega \Gamma \sin \theta}{z_{o} C} z\right) \sin 2 \theta+i \frac{d_{F}}{2 \Gamma \sin \theta} \cos 2 \theta-\frac{d_{F}}{2 \Gamma \sin \theta} \sin 2 \theta
\end{aligned}
$$

where:

$$
d_{F}=\frac{\frac{3}{4} \beta|\varepsilon(0)|^{2} \Gamma^{4}}{\gamma}
$$

Thus the forward wave inside the resonator $E_{F}(z, t)$ can be written in the form:

$$
E_{F}(z, t)=\varepsilon_{F}(0) \exp \left(-\frac{\alpha_{F} z}{2}\right) \exp i\left(\omega t-k_{F} z\right)
$$

where:

$$
\alpha_{F}=\frac{\omega}{\varepsilon_{0} C} \Gamma \sin \theta-\frac{d_{F}}{z \Gamma \sin \theta} \sin 2 \theta\left(1-\exp \left(-\frac{\omega \Gamma \sin \theta}{\varepsilon_{0} C} z\right)\right)
$$

and

$$
k_{F}=\frac{\omega}{C}\left[1+\frac{\Gamma \cos \theta}{2 \varepsilon_{o}}-\frac{d_{F}}{2 z \Gamma \sin \theta} \cos 2 \theta \frac{C}{\omega}\left(1-\exp \left(-\frac{\omega \Gamma \sin \theta}{\varepsilon_{0} C} z\right)\right)\right]
$$

There are two terms determine the value of the absorption coefficient $\alpha_{F}$, as shown above. The first term is independent of the intensity of the field while the second term depends on the field intensity.

The absorption coefficient should be low enough to sufficient number of reflected beams inside the resonator to happen .These reflections will provide sufficient feedback for the existence of the bistable phenomena. For more explanation let us consider the following example. 
Consider the medium inside the resonator has an absorption coefficient $\alpha$ and thickness L. The reflection coefficient of the resonator's mirrors is $\mathrm{R}$. Then, the number of effective reflected beams will be $\frac{1}{1-\operatorname{Re} x p\left(-\alpha_{F} L\right)}$. For number of beam reflections of 10 , while $\mathrm{R}=0.99$ then $\alpha \mathrm{L}$ should be 0.095 . Thus we can consider $\frac{\omega \Gamma \sin \theta}{\varepsilon_{0} C} \mathrm{Z} \ll<1$, therefore, we get

$$
\alpha_{F} \approx \frac{\omega}{\varepsilon_{0} C} \Gamma \sin \theta\left[1-\frac{2 d_{F} \cos \theta}{\Gamma}\right]
$$

the above expression for $\alpha_{F}$ can be shortened and get its absolute value as,

$$
\alpha_{F}=\frac{\alpha_{0}}{1+\frac{I_{c}}{I_{s}}}
$$

with:

$$
\begin{aligned}
& \alpha_{0}=\frac{\omega \Gamma \sin \theta}{\varepsilon_{0} C}=\frac{2 \omega^{2} \sigma \gamma}{\varepsilon_{0} C\left[\left(\omega_{0}^{2}-\omega^{2}\right)^{2}+4 \sigma^{2} \omega^{2}\right]}\left(\mathrm{cm}^{-1}\right) \\
& \mathrm{I}_{\mathrm{C}}=\frac{1}{2} \varepsilon_{0} c\left|\varepsilon_{F}(0)\right|^{2}\left(\mathrm{~W} / \mathrm{cm}^{2}\right) \\
& \mathrm{I}_{\mathrm{s}}=\frac{1}{4} \varepsilon_{0} c \frac{\gamma}{\Gamma^{3} \beta \cos \theta}\left(\mathrm{W} / \mathrm{cm}^{2}\right) \\
& \mathrm{I}_{\mathrm{s}}=\frac{1}{4} \varepsilon_{0} c \frac{\left[\left(\omega_{0}^{2}-\omega^{2}\right)^{2}+4 \delta^{2} \omega^{2}\right]^{2}}{\gamma^{2} \beta\left(\omega_{0}^{2}-\omega^{2}\right)}\left(\mathrm{W} / \mathrm{cm}^{2}\right)
\end{aligned}
$$

and:

$$
\mathrm{k}_{\mathrm{F}}=\frac{\omega}{C}\left[1+\frac{\gamma\left(\omega_{0}^{2}-\omega^{2}\right)}{2 \varepsilon_{0}\left\{\left(\omega_{0}^{2}-\omega^{2}\right)^{2}+4 \sigma^{2} \omega^{2}\right\}}-\frac{\frac{3}{4} \beta \gamma^{3}\left\{\left(\omega_{0}^{2}-\omega^{2}\right)^{2}-4 \sigma^{2} \omega^{2}\right\}\left|\mathcal{E}_{\omega}^{F}(0)\right|^{2}}{2 \varepsilon_{0}\left\{\left(\omega_{0}^{2}-\omega^{2}\right)^{2}+4 \sigma^{2} \omega^{2}\right)^{3}}\right]
$$

where:

$\mathrm{I}_{\mathrm{C}} \quad$ is the intensity inside the laser cavity

$\mathrm{I}_{\mathrm{s}}$ is the saturation intensity (atomic constant)

$\mathrm{K}$ is the wave vector 
Similarly the backward field is given by:

$$
E_{B}(z, t)=\varepsilon_{B}(0) \exp \left(-\frac{\alpha_{B}(L-z)}{2}\right) \operatorname{expi}\left(\omega \mathrm{t}-k_{B}(\mathrm{~L}-\mathrm{z})\right)
$$

where:

$$
\alpha_{B}=\frac{\omega}{\varepsilon_{0} C} \Gamma \sin \theta-\frac{d_{B}}{(L-Z) \Gamma \sin \theta} \sin 2 \theta\left(1 \exp \left(-\frac{\omega \Gamma \sin \theta}{\varepsilon_{0} C}(L-z)\right)\right.
$$

and:

$$
\mathrm{k}_{\mathrm{B}}=\frac{\omega}{C}\left[1+\frac{\Gamma \cos \theta}{2 \varepsilon_{0}}-\frac{d_{B}}{2(L-z) \Gamma \sin \theta} \cos 2 \theta \frac{C}{\omega}\left(1-\exp \left(-\frac{\omega \Gamma \sin \theta}{\varepsilon_{0} C}(L-z)\right)\right]\right.
$$

The boundary conditions of the fields inside the resonator are:

$$
\begin{aligned}
& \mathrm{E}_{\mathrm{F}}(0, \mathrm{t})=\sqrt{T} \mathrm{E}_{\mathrm{I}}(0, \mathrm{t})+\sqrt{R} \mathrm{E}_{\mathrm{B}}(0, \mathrm{t}) \\
& \mathrm{E}_{\mathrm{T}}(\mathrm{L}, \mathrm{t})=\sqrt{T} \mathrm{E}_{\mathrm{F}}(\mathrm{L}, \mathrm{t}) \\
& \mathrm{E}_{\mathrm{B}}(\mathrm{L}, \mathrm{t})=\sqrt{R} \mathrm{E}_{\mathrm{F}}(\mathrm{L}, \mathrm{t}) \\
& \mathrm{E}_{\mathrm{B}}(\mathrm{z}, \mathrm{t})=\mathrm{E}_{\mathrm{B}}(\mathrm{L}, \mathrm{t}) \exp \left(-\alpha_{B}(L-z)\right) \exp \left(-2 i k_{B}(L-z)\right)
\end{aligned}
$$

where:

$$
\begin{array}{ll}
\mathrm{T} & \text { is the transmission coefficient } \\
\mathrm{R} & \text { is the reflection coefficient }
\end{array}
$$

From (32) and (35) one gets:

$$
\begin{aligned}
\mathrm{E}_{\mathrm{B}}(0, \mathrm{t})= & \mathrm{E}_{\mathrm{B}}(\mathrm{L}, \mathrm{t}) \exp \left(-\frac{\alpha_{B}}{2} L\right) \exp \left(-i k_{B} L\right)=\sqrt{R} \mathrm{E}_{\mathrm{F}}(\mathrm{L}, \mathrm{t}) \exp \left(-\frac{\alpha_{B}}{2} L\right) \\
& \exp \left(-i k_{B} L\right)
\end{aligned}
$$

Through the boundary conditions and equations (33) and (37) one gets:

$$
\begin{aligned}
& \mathrm{E}_{\mathrm{F}}(0, \mathrm{t})=\sqrt{T} \mathrm{E}_{\mathrm{I}}(0, \mathrm{t})+\mathrm{R} \mathrm{E}_{\mathrm{F}}(\mathrm{L}, \mathrm{t}) \exp \left(-\frac{\alpha_{B}}{2} L\right) \exp \left(-i k_{B} L\right) \\
& \mathrm{E}_{\mathrm{F}}(0, \mathrm{t})=\sqrt{T} \mathrm{E}_{\mathrm{I}}(0, \mathrm{t})+\frac{R}{\sqrt{T}} \mathrm{E}_{\mathrm{T}}(\mathrm{L}, \mathrm{t}) \exp \left(-\frac{\alpha_{B}}{2} L\right) \exp \left(-i k_{B} L\right)
\end{aligned}
$$


Considering equation (34) and the above obtained results it follows:

$\mathrm{E}_{\mathrm{F}}(\mathrm{z}, \mathrm{t})=\left[\sqrt{T} \mathrm{E}_{\mathrm{I}}(0, \mathrm{t})+\frac{R}{\sqrt{T}} \mathrm{E}_{\mathrm{T}}(\mathrm{L}, \mathrm{t}) \exp \left(-\frac{\alpha_{B}}{2} L\right) \exp \left(-i k_{B} L\right)\right] \exp \left(-\frac{\alpha_{F}}{2} z\right)$

$$
\exp \left(-i k_{F} z\right)
$$

$\mathrm{E}_{\mathrm{T}}(\mathrm{L}, \mathrm{t})=\sqrt{T}\left[\sqrt{T} \mathrm{E}_{\mathrm{I}}(0, \mathrm{t})+\frac{R}{\sqrt{T}} \mathrm{E}_{\mathrm{T}}\right.$

$$
\begin{gathered}
\left.(\mathrm{L}, \mathrm{t}) \exp \left(-\frac{\alpha_{B}}{2} L\right) \exp \left(-i k_{B} L\right)\right] \exp \left(-\frac{\alpha_{F}}{2} L\right) \exp \left(-i k_{F} L\right) \\
\mathrm{E}_{\mathrm{T}}(\mathrm{L}, \mathrm{t})=\frac{T E_{I}(0, t) \exp \left(-\frac{\alpha_{F}}{2} L\right) \exp \left(-i k_{F} L\right)}{1-\operatorname{Re} \operatorname{xp}\left(-\frac{\alpha_{F}+\alpha_{B}}{2} L\right) \exp -i\left(k_{F}+k_{B}\right) L} \\
\mathrm{I}_{\mathrm{T}}=\left|\mathrm{E}_{\mathrm{T}}(\mathrm{L}, \mathrm{t})\right|^{2}= \\
\frac{T^{2} I_{i n} \exp \left(-\alpha_{F} L\right)}{1-2 \operatorname{Re} \operatorname{xp}\left(-\frac{\alpha_{F}+\alpha_{B}}{2} L\right) \cos \left(k_{F}+k_{B}\right) L+R^{2} \exp -\left(\alpha_{F}+\alpha_{B}\right) L}
\end{gathered}
$$

To get $\left(\mathrm{I}_{\text {cavity }}\right)$ :

$$
\begin{gathered}
\varepsilon_{\text {cavity }}(\mathrm{z}, \mathrm{t})=\varepsilon_{F}(z, t)+\varepsilon_{B}(z, t) \\
\varepsilon_{\text {cavity }}(z, t)=\frac{\sqrt{T} E_{I}(0, t) \exp \left(-\frac{\alpha_{F}}{2} z\right) \exp \left(-i k_{F} z\right)}{1-\operatorname{Rexp}\left(-\frac{\alpha_{F}+\alpha_{B}}{2} L\right) \exp -i\left(k_{F}+k_{B}\right) L} \\
+\frac{\alpha_{F} \sqrt{R} E_{I}(0, t) \exp \left(-\frac{\alpha_{F}}{2} L\right) \exp \left(-i k_{F} L\right) \exp \left(-\frac{\alpha_{B}}{2}(L-Z)\right) \exp \left(-i k_{B}(L-z)\right)}{1-\operatorname{Re} x p\left(-\frac{\alpha_{F}+\alpha_{B}}{2} L\right) \exp -i\left(k_{F}+k_{B}\right) L} \exp (i \phi)
\end{gathered}
$$

where: $\phi$ is the phase gained by backward wave.

$$
\mathrm{I}_{\text {cavity }}=
$$

$\frac{I_{\text {in }} T\left(\exp \left(-\alpha_{F} z\right)+\operatorname{Re} x p\left(-\alpha_{F} L\right) \exp \left(-\alpha_{B}(L-z)\right)+2 \sqrt{R} \exp \left(-\frac{\alpha_{F}}{2}(L+z)\right) \exp \left(-\frac{\alpha_{B}}{2}(L-z)\right) \operatorname{Cos}\left(\left(K_{F}+K_{B}\right)(L-z)\right)\right\}}{1-2 \operatorname{Re} x p\left(-\frac{\alpha_{F}+\alpha_{B}}{2} L\right) \cos \left(k_{F}+k_{B}\right) L+R^{2} \exp -\left(\alpha_{F}+\alpha_{B}\right) L}$

For $\mathrm{R} \leq 1$, consider $\alpha_{F}=\alpha_{B}$ and $k_{F}=k_{B}$ 
$\mathrm{I}_{\text {cavity }}=$

$\frac{I_{i n} T\left\{\exp \left(-\alpha_{F} z\right)+\operatorname{Re} x p\left(-\alpha_{F} L\right) \exp \left(-\alpha_{B}(L-z)\right)+2 \sqrt{R} \exp \left(-\frac{\alpha_{F}}{2} 2 L\right) \cos (\phi) \operatorname{Cos}\left(2 K_{F}(L-z)\right)\right\}}{1-2 \operatorname{Re} x p\left(-\alpha_{F} L\right) \cos \left(2 k_{F}\right) L+R^{2} \exp -\left(2 \alpha_{F}\right) L}$

Taking the average the average over $\mathrm{z}$ we get,

$\mathrm{I}_{\text {cavity }}=$

$$
I_{i n} T \frac{\left.\frac{1}{\alpha_{F} L}\left(1-\exp \left(-\alpha_{F} L\right)\right)\left(1+\operatorname{Re} \operatorname{xp}\left(-\alpha_{F} L\right)\right)+2 \sqrt{R} \exp \left(-\alpha_{F} L\right) \cos (\phi)\right)}{1-2 \operatorname{Re} x p\left(-\alpha_{F} L\right) \cos \left(2 k_{F} L\right)+R^{2} \exp -\left(2 \alpha_{F} L\right)}
$$

\section{Case (1): for a Lorentz spectral line profile}

$$
\mathrm{I}_{\mathrm{T}}=\int_{-\infty}^{\infty} E_{T}(L, t) E_{T}^{*}(L, t) I_{L} d \omega
$$

where:

$$
\begin{gathered}
I_{L}=\frac{1}{1+\left(\frac{v-v_{0}}{\Delta v / 2}\right)^{2}} \frac{2}{\pi \Delta v} \text { is Lorentz distribution. } \\
\therefore \quad \mathrm{I}_{\mathrm{T}}=\mathrm{T}^{2} \mathrm{I}_{\text {in }} \\
\exp \left(-\alpha_{F} L\right) \int_{-\infty}^{\infty} \frac{I_{L}}{1-2 \operatorname{Re} x p(-\alpha L) \cos k L+R^{2} \exp (-2 \alpha L)} d \omega \\
\text { let } \mathrm{A}=1+\mathrm{R}^{2} \exp (-2 \alpha L), \text { and } \mathrm{B}=2 \mathrm{R} \exp (-\alpha L)
\end{gathered}
$$

where:

$$
\alpha=\frac{\alpha_{F}+\alpha_{B}}{2} \quad \& \quad \mathrm{k}=\mathrm{k}_{\mathrm{F}}+\mathrm{k}_{\mathrm{B}}
$$

Then, $\quad \mathrm{I}_{\mathrm{T}}=\mathrm{T}^{2} \mathrm{I}_{\mathrm{in}} \exp \left(-\alpha_{F} L\right) \int_{-\infty}^{\infty} \frac{I_{L}}{A-B \cos \left(2 \operatorname{Ln} \frac{2 \pi}{C} v\right)} d \omega$

where:

$$
\mathrm{n}=\frac{n_{F}+n_{B}}{2}
$$

and due to the complexity of integration (41), we choose another equivalent relation that can be integrated easily,

$$
\frac{1}{A-B \cos \left(2 \operatorname{Ln} \frac{2 \pi v}{C}\right)}=\frac{1 /(A-B)}{1+\left(\frac{v-v_{r}}{\delta v / 2}\right)^{2}}
$$


where:

$$
\begin{gathered}
\delta v=\frac{C}{2 \pi L n} \frac{1-\mathrm{Re}^{-\alpha L}}{\sqrt{\operatorname{Re}^{-\alpha L}}} \quad \& \quad v_{r}=\frac{m C}{2 L n} \\
\int_{-\infty}^{\infty} \frac{I_{L}}{A-B \cos \left(2 \operatorname{Ln} \frac{2 \pi}{C} v\right)} d \omega=\int_{-\infty}^{\infty} \frac{2 / \pi \Delta v}{1+\left(\frac{v-v_{0}}{\Delta v / 2}\right)^{2}} \frac{1 /(A-B)}{1+\left(\frac{v-v_{r}}{\delta v / 2}\right)^{2}} d v
\end{gathered}
$$

Equation (42) can be written in the form:

$$
\begin{aligned}
& \int_{-\infty}^{\infty} \frac{2 / \pi \Delta v}{1+\left(\frac{v-v_{0}}{\Delta v / 2}\right)^{2}} \frac{1 /(A-B)}{1+\left(\frac{v-v_{r}}{\delta v / 2}\right)^{2}} d v= \\
& \frac{2(\delta v / 2)^{2}(\Delta v / 2)^{2}}{\pi(\Delta v)(A-B)} \int_{-\infty}^{\infty} \frac{1}{a_{1} v^{2}+b_{1} v+c_{1}} \frac{1}{a_{2} v^{2}+b_{2} v+c_{2}} d v
\end{aligned}
$$

where: $\quad a_{1}=1, \mathrm{~b}_{1}=-2 v_{0}$ and $\mathrm{c}_{1}=(\Delta v / 2)^{2}+v_{0}^{2}$

$$
a_{2}=1, \mathrm{~b}_{2}=-2 v_{r} \text { and } \mathrm{c}_{2}=(\delta v / 2)^{2}+v_{r}^{2}
$$

Using partial fraction,

$$
\begin{aligned}
& \frac{2(\delta v / 2)^{2}(\Delta v / 2)^{2}}{\pi(\Delta v)(A-B)} \int_{-\infty}^{\infty} \frac{1}{a_{1} v^{2}+b_{1} v+c_{1}} \frac{1}{a_{2} v^{2}+b_{2} v+c_{2}} d v \\
& =\frac{2(\delta v / 2)^{2}(\Delta v / 2)^{2}}{\pi(\Delta v)(A-B)} \int_{-\infty}^{\infty} \frac{A_{1} v+B_{1}}{a_{1} v^{2}+b_{1} v+c_{1}}+\frac{A_{2} v+B_{2}}{a_{2} v^{2}+b_{2} v+c_{2}} d v
\end{aligned}
$$

To get the coefficients $\mathrm{A}_{1}, \mathrm{~A}_{2}, \mathrm{~B}_{1}$ and $\mathrm{B}_{2}$, we make the following:

$$
\left(A_{1} v+B_{1}\right)\left(a_{2} v^{2}+b_{2} v+c_{2}\right)+\left(A_{2} v+B_{2}\right)\left(a_{1} v^{2}+b_{1} v+c_{1}\right)=1
$$

and equating the coefficient of $v^{3}, v^{2}, v^{1}, v^{0}$, we get:

$$
\begin{aligned}
& a_{2} A_{1}+a_{1} A_{2}=0 \\
& b_{2} A_{1}+a_{2} B_{1}+b_{1} A_{2}+a_{1} B_{2}=0
\end{aligned}
$$




$$
\begin{aligned}
& c_{2} A_{1}+b_{2} B_{1}+c_{1} A_{2}+b_{1} B_{2}=0 \\
& c_{2} B_{1}+c_{1} B_{2}=1
\end{aligned}
$$

In case $\mathrm{b}_{1}=\mathrm{b}_{2}$ or $v_{0}=v_{r}$ then:

$$
\begin{aligned}
\mathrm{A}_{1} & =\mathrm{A}_{2}=0 \quad \& \quad \mathrm{~B}_{1}=-\mathrm{B}_{2}=- \\
\frac{1}{c_{1}-c_{2}} & =-\frac{1}{(\Delta v / 2)^{2}-(\delta v / 2)^{2}}
\end{aligned}
$$

Since $a_{1}=a_{2}=1$, then:

$$
\begin{aligned}
& \frac{2(\delta v / 2)^{2}(\Delta v / 2)^{2}}{\pi(\Delta v)(A-B)} \int_{-\infty}^{\infty} \frac{B_{1}}{v^{2}+b_{1} v+c_{1}}+\frac{B_{2}}{v^{2}+b_{2} v+c_{2}} d v= \\
& \frac{2(\delta v / 2)^{2}(\Delta v / 2)^{2}}{\pi(\Delta v)(A-B)} \int_{-\infty}^{\infty} \frac{B_{1}}{v^{2}+b_{1} v+c_{1}} d v+\frac{2(\delta v / 2)^{2}(\Delta v / 2)^{2}}{\pi(\Delta v)(A-B)} \\
& \int_{-\infty}^{\infty} \frac{B_{2}}{v^{2}+b_{2} v+c_{2}}= \\
& 2 \frac{2(\delta v / 2)^{2}(\Delta v / 2)^{2}}{\pi(\Delta v)(A-B)} \int_{0}^{\infty} \frac{B_{1}}{v^{2}+b_{1} v+c_{1}}+\frac{B_{2}}{v^{2}+b_{2} v+c_{2}} d v= \\
& \frac{2(\delta v / 2)^{2}(\Delta v / 2)^{2}}{\pi(\Delta v)(A-B)}\left[(\pi / 2)\left\{\frac{2 B_{1}}{\sqrt{4 c_{1}-b_{1}^{2}}}+\frac{2 B_{2}}{\sqrt{4 c_{2}-b_{2}^{2}}}\right\}-\right. \\
& \left.\left\{\frac{2 B_{1}}{\sqrt{4 c_{1}-b_{1}^{2}}} \arctan \frac{b_{1}}{\sqrt{4 c_{1}-b_{1}^{2}}}+\frac{2 B_{2}}{\sqrt{4 c_{2}-b_{2}^{2}}} \arctan \frac{b_{2}}{\sqrt{4 c_{2}-b_{2}^{2}}}\right\}\right] .
\end{aligned}
$$

Then, $\mathrm{I}_{\mathrm{T}}=\left[2 \frac{T^{2} I_{\text {in }} \exp (-\alpha L)}{\left(1-\operatorname{Re} x p(-\alpha L)^{2}\right.} \frac{2}{\pi}\left(\frac{\delta v}{2}\right)^{2}\right] \times$

$$
\left[\begin{array}{l}
\frac{\pi}{2}\left(\frac{-1}{(\Delta v / 2)^{2}-(\delta v / 2)^{2}}+\frac{\Delta v / 2}{\delta v / 2\left[(\Delta v / 2)^{2}-(\delta v / 2)^{2}\right]}\right)+ \\
\left(\frac{-1}{(\Delta v / 2)^{2}-(\delta v / 2)^{2}} \arctan \left(\frac{-v_{0}}{\Delta v / 2}\right)+\frac{\Delta v / 2}{\delta v / 2\left[(\Delta v / 2)^{2}-(\delta v / 2)^{2}\right.} \arctan \frac{-v_{0}}{\delta v / 2}\right.
\end{array}\right]
$$




\section{Case (2): for Gaussian distribution:}

$$
\exp (-\alpha L) \int_{-\infty}^{\infty} \frac{\mathrm{I}_{\mathrm{T}}=\mathrm{T}^{2} \mathrm{I}_{\text {in }}}{I_{G}-2 \operatorname{Re} x p(-\alpha L) \cos \left(2 \operatorname{Ln} \frac{2 \pi}{C} v\right)+R^{2} \exp (-2 \alpha L)} d v
$$

where:

$$
\mathrm{I}_{\mathrm{G}}=\sqrt{\frac{4 \ln 2}{\pi(\Delta v)^{2}}} \exp \left(-4 \ln 2\left(\frac{v-v_{0}}{\Delta v}\right)^{2}\right) \quad \text { is Gaussian distribution. }
$$

Thus: ${ }_{\mathrm{T}}=\mathrm{T}^{2} \mathrm{I}_{\mathrm{in}} \exp (-\alpha L)$

$\sqrt{\frac{4 \ln 2}{\pi(\Delta v)^{2}}} \int_{-\infty}^{\infty} \frac{\exp \left(-\tau x^{2}\right)}{1-2 a \cos \left(2 \operatorname{Ln} \frac{2 \pi}{C}\left(x+v_{0}\right)\right)+a^{2}} d x$

$$
\text { where: }-v_{0}=\mathrm{x}, \quad a=\mathrm{R} \exp (-\alpha L) \quad \& \quad \tau=\frac{4 \ln 2}{(\Delta v)^{2}}
$$

$\omega_{0}$ is considered to be a resonance frequency of the Febry-Perot resonator.

$$
\mathrm{I}_{\mathrm{T}}=\mathrm{T}^{2} \mathrm{I}_{\text {in }} \exp (-\alpha L) \sqrt{\frac{4 \ln 2}{\pi(\Delta v)^{2}}} \frac{1}{k} \int_{-\infty}^{\infty} \frac{\exp \left(-\left(\tau / k^{2}\right) y^{2}\right)}{1-2 a \cos y+a^{2}} d y
$$

where:

$$
\begin{aligned}
& (2 \mathrm{~nL} / \mathrm{c}) \mathrm{x}=\mathrm{kx}=\mathrm{y}, \quad \mathrm{dx}=\mathrm{dy} / \mathrm{k}, \quad \text { and } \quad \frac{\tau}{k^{2}}=\rho \\
& \mathrm{I}_{\mathrm{T}}=\mathrm{T}^{2} \mathrm{I}_{\text {in }} \exp (-\alpha L) \sqrt{\frac{4 \ln 2}{\pi(\Delta v)^{2}}} \frac{1}{k} \int_{-\infty}^{\infty} \frac{\exp \left(-(\rho) y^{2}\right)}{1-2 a \cos y+a^{2}} d y \\
& \mathrm{I}_{\mathrm{T}}=\mathrm{T}^{2} \mathrm{I}_{\text {in }} \exp (-\alpha L) \sqrt{\frac{4 \ln 2}{\pi(\Delta v)^{2}}} \frac{1}{k} \frac{\sqrt{\frac{\pi}{\rho}}}{1-a^{2}}\left\{\frac{1}{2}+\sum_{i=1}^{\infty} a^{i} \exp \left(-\frac{i^{2}}{4 \rho}\right)\right\} \\
& \mathrm{I}_{\mathrm{T}}=2 \frac{T^{2} I_{i n} \exp (-\alpha L)}{1-R^{2} \exp (-2 \alpha L)}\left\{\frac{1}{2}+\sum_{i=1}^{\infty}(\operatorname{Re} \operatorname{xp}(-\alpha L))^{i} \exp \left(-\frac{i^{2} k^{2}(\Delta v)^{2}}{16 \ln 2}\right)\right.
\end{aligned}
$$


To get $I_{\text {cavity }}$ for these two cases:

$$
\begin{aligned}
& I_{\text {cavity }}^{G}=\int_{-\infty}^{\infty} I_{i n} T\left\{\frac{\frac{1}{\alpha L}\left(1-\exp \left(-\alpha_{F} L\right)\right)\left(1+\operatorname{Re} \exp \left(-\alpha_{F} L\right)\right)+2 \sqrt{R} \exp \left(-\alpha_{F} L\right) \cos (\phi)}{1-2 \operatorname{Rexp}\left(-\alpha_{F} L\right) \operatorname{Cos}(\delta)+R^{2} \exp \left(-2 \alpha_{F} L\right)}\right\} \times I_{G} d v \\
& I_{\text {cavity }}^{L}=\int_{-\infty}^{\infty} I_{i n} T\left\{\frac{\frac{1}{\alpha L}\left(1-\exp \left(-\alpha_{F} L\right)\right)\left(1+\operatorname{Rexp}\left(-\alpha_{F} L\right)\right)+2 \sqrt{R} \exp \left(-\alpha_{F} L\right) \cos (\phi)}{1-2 \operatorname{Rexp}\left(-\alpha_{F} L\right) \operatorname{Co}(\delta)+R^{2} \exp \left(-2 \alpha_{F} L\right)}\right\} \times I_{G} d v
\end{aligned}
$$

where:

$$
\begin{aligned}
& I_{L}=\frac{1}{1+\left(\frac{v-v_{0}}{\Delta v / 2}\right)^{2}} \frac{2}{\pi \Delta v} \text { is Lorentz distribution. } \\
& \mathrm{I}_{\mathrm{G}}=\sqrt{\frac{4 \ln 2}{\pi(\Delta \omega)^{2}}} \exp \left(-4 \ln 2\left(\frac{\varpi-\varpi_{0}}{\Delta \varpi}\right)^{2}\right. \text { is Gaussian distribution } \\
& I_{\text {cavity }}^{G}=I_{T}^{G}\left\{\frac{\frac{1}{\alpha L}(1-\exp (-\alpha L))(1+\operatorname{Re} \operatorname{xp}(-\alpha L))+2 \sqrt{R} \exp (-\alpha L) \cos (\phi)}{T \exp (-\alpha L)}\right\} \\
& I_{\text {cavity }}^{L}=I_{T}^{L}\left\{\frac{\frac{1}{\alpha L}(1-\exp (-\alpha L))(1+\operatorname{Re} \operatorname{xp}(-\alpha L))+2 \sqrt{R} \exp (-\alpha L) \cos (\phi)}{T \exp (-\alpha L)}\right\}
\end{aligned}
$$

\section{Calculations:}

From Equations (25), (43), (44) and (45) assuming a value for $\alpha_{o} L$ and given values for $\frac{I_{c}}{I_{s}}$ corresponding values for $\frac{I_{i n}}{I_{s}}$ are calculated from Equation (45), after dividing its both sides by $I_{s}$. Substituting in Equation (43) and (44), after dividing its both sides by $I_{s}$, for the values of $\frac{I_{c}}{I_{s}}$ and the corresponding 
calculated values of $\frac{I_{\text {in }}}{I_{s}}, \frac{I_{\text {out }}}{I_{s}}$ can be calculated. The calculations are carried out for $R=0.99$, and $\alpha_{o} L=0.2,0.5$ and $0.7 . \frac{I_{c}}{I_{s}}$ is given values from 0.0 up to 50 in steps of 0.05 leading to varying the nonlinear absorption coefficient $\alpha$. The behavior of the relative intensities of $\frac{I_{\text {in }}}{I_{s}}$ against $\frac{I_{\text {out }}}{I_{s}}$ is shown in figures. The phase shift $\phi$ suffered by the backward wave with respect to the forward one is considered to take the value $\pi$.

The above figures can carried out for half width range from $0,0.2 \times 10^{8}$ to $1.2 \times 10^{8} \mathrm{~Hz}$ in step of 0.2 .Figures (1, 2 and 3) show the behavior of $\frac{I_{\text {out }}}{I_{s}}$ against $\frac{I_{\text {in }}}{I_{s}}$ for the case of Gaussian line profile. Figures $(4,5$ and 6$)$ are the corresponding figures to figures (1,2 and 3 ) of the Lorentzian line profile.

\section{Discussion:}

From the Figures (1- 6), we observe that:

The bistability phenomena are more pronounced as the spectral half width of the interacting field with the medium is decreased. It can be attributed to the bistability phenomena that arise through interference phenomena which requires temporal coherent interacting field and in turn a field of small spectral half width.

The Interacting fields of Gaussian line profile are more effective for initiating bistability than a field of Lorentz profile. It is due to the fact that effective spectral range is in case of Lorentz profile greater than that in case of Gaussian profile.

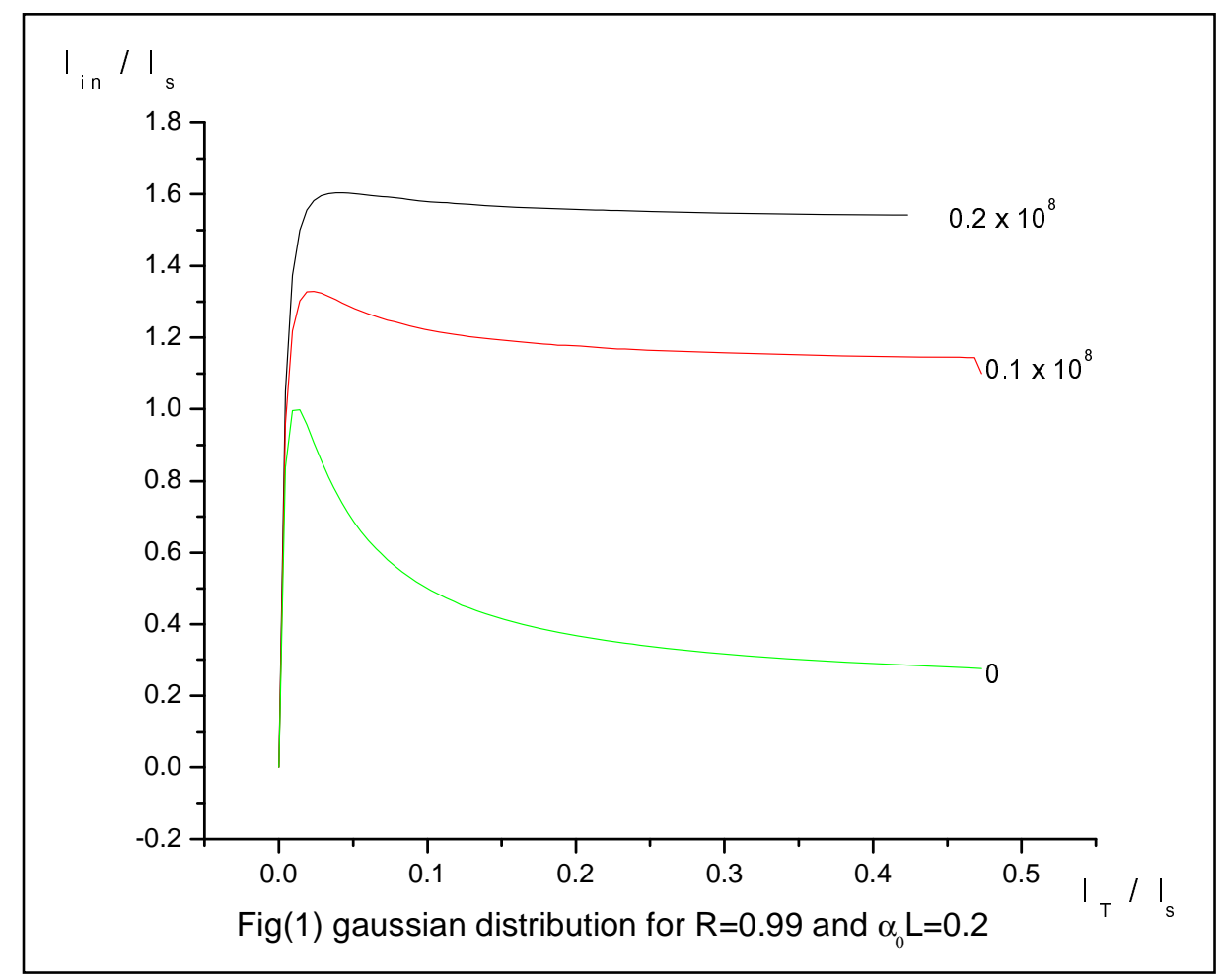



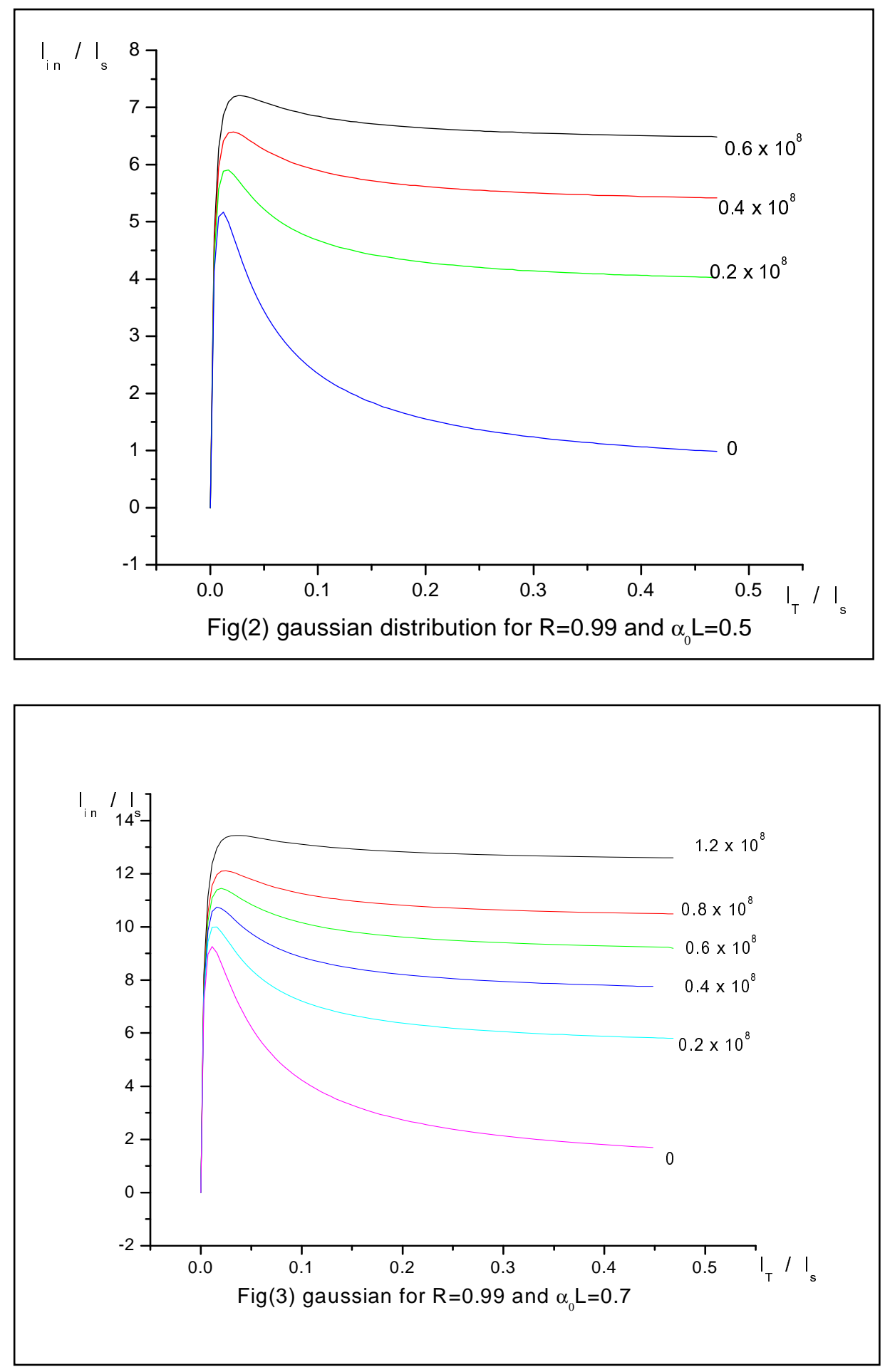


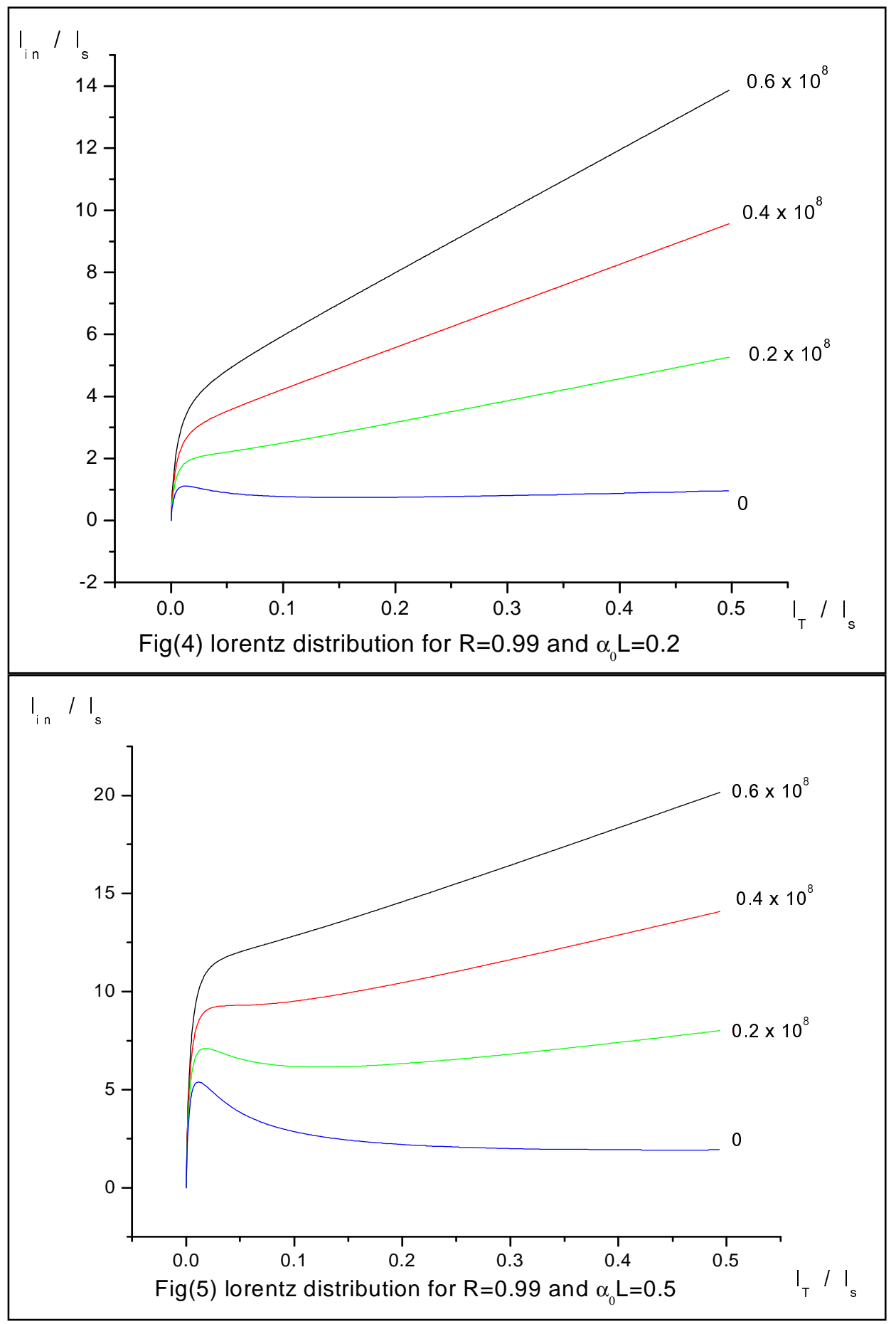




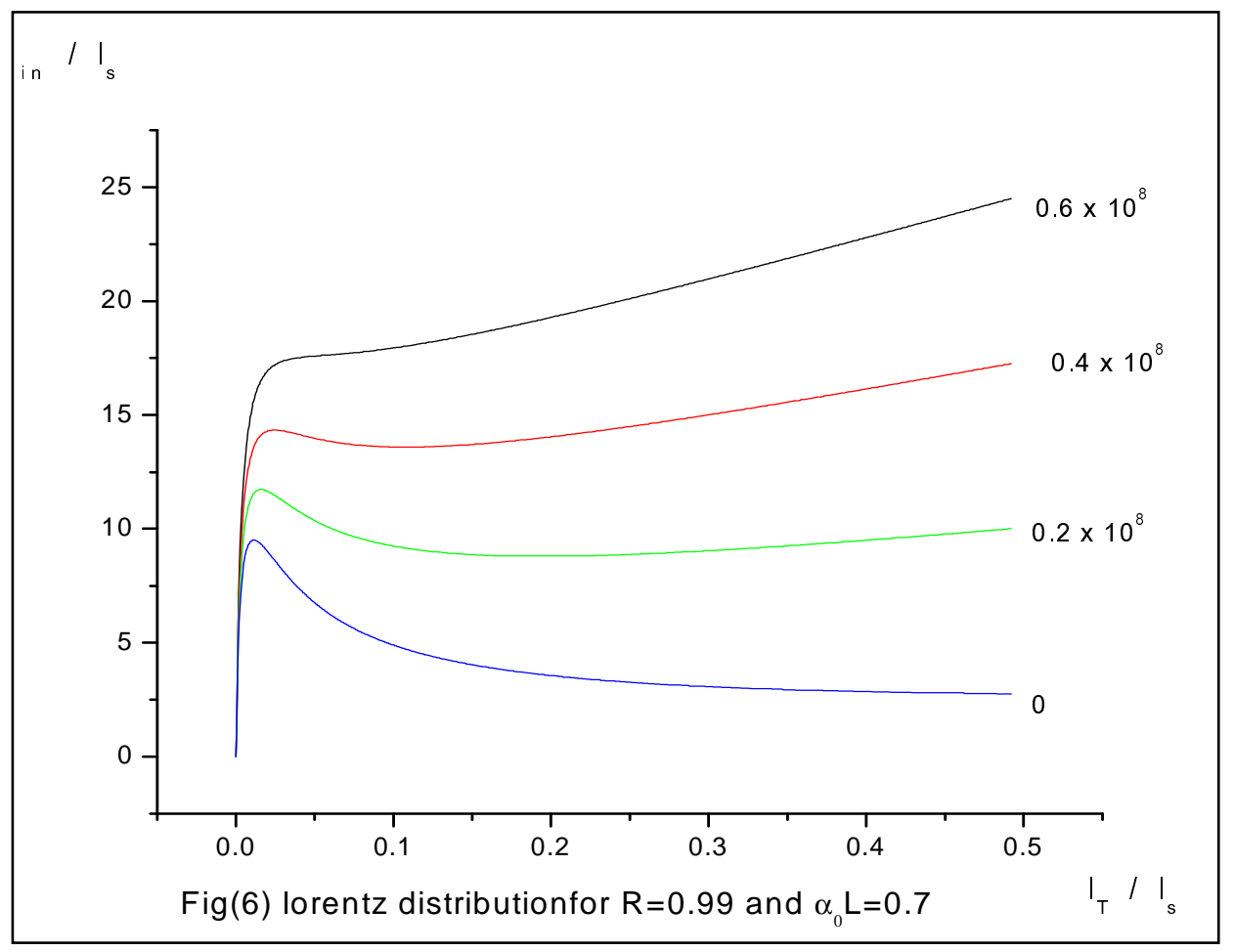

There are two parameters controlling the bistability, the reflection coefficient $\mathrm{R}$ and the nonlinear absorption coefficient $\alpha$ [15]. $\mathrm{R}$ is responsible for the interference phenomena and also for increasing the life time of the interacting photon inside the resonator, which in turn leading to increase the probability of interaction of the photon with the medium. On the other hand, $\alpha$ is responsible for a direct interaction of the photon with the medium.

We can conclude that to get bistability it is sufficient that either $\alpha$ must be large enough and $\mathrm{R}$ can be of small values or vice versa.

\section{References:}

1. T. H. Maiman, Nature, 187, 493 (1960).

2. Franken, A. E. Hill, C. W. Peters and G. Weinreich, Phys. Rev. lett. 7, 118 (1961).

3. W. Kaiser and G. C. B. Garrett, Phys. Rev. Lett. 7, 229, 231(1961).

4. E. J. Woodburg and W. K. Ng, Proc. IEEE 50, 2367(1962).

5. J. A. Giordamaine, Phys. Rev. Lett. (8), 19-20 (1962). 
6. P. D. Maker, R. W. Terhune, M. Nisenhoff, and C. M. Savage, Phys. Rev. Lett. (8) 21-22 (1962).

7. P. D. Maker, R. W. Terhune, and C. M. Savage, "Quantum Electronics", edited by P. Grivet and N. Bloembergen (Colombia University, New York, 1964) p. 1559.

8. P. D. Maker and R. W. Terhune, Phys. Rev. A137, 801 (1965).

9. A. Yariv, Principles of Quantum Electronics, 2nd ed., Wiley New York, (1975).

10. P. W. Smith and W. J. Tomlinson, IEEE Spectrum, 8 (6), 26 (1981).

11. S. Lynch, A. L. Steelf and J. E. Hoad, Chaos, Solitons and Fractals 9 (6), 935 (1998).

12. N. Agishev, N. A. Ivanova and A. L. Tolstik, Optics Communications 156, 199 (1998).

13. Khian-Hooichew, Junaidah Osman and David Reginald Tilley, "The Nonlinear Fabry- Perot Resonator Direct Numerical Integration", Optics Communications 191, 393 (2001).

14. M. M. El-Nicklawy, A. F. Hassan, S. M. M. Salman, and A. Abdel-Aty, Optics and Laser Technology, 34, 363 (2002).

15. M. M. El-Nicklawy, A. F. Hassan, S. M. M. Salman, and A. Abdel-Aty, Optics and Laser Technology, 37, 363 (2005). 